\title{
Diffusive hydrodynamics from integrability breaking
}

\author{
Aaron J. Friedman ${ }^{1,2}$, Sarang Gopalakrishnan ${ }^{3}$, and Romain Vasseur ${ }^{2}$ \\ ${ }^{1}$ Department of Physics and Astronomy, University of California, Irvine, CA 92697, USA \\ ${ }^{2}$ Department of Physics, University of Massachusetts, Amherst, Massachusetts 01003, USA \\ ${ }^{3}$ Department of Physics and Astronomy, CUNY College of Staten Island, \\ Staten Island, NY 10314; Physics Program and Initiative for the Theoretical Sciences, \\ The Graduate Center, CUNY, New York, NY 10016, USA
}

\begin{abstract}
We describe the crossover from generalized to conventional hydrodynamics in nearly integrable systems. Integrable systems have infinitely many conserved quantities, which spread ballistically in general. When integrability is broken, only a few of these conserved quantities survive. The remaining conserved quantities are generically transported diffusively; we derive a compact and general diffusion equation for these. The diffusion constant depends on the matrix elements of the integrability-breaking perturbation; for a certain class of integrability-breaking perturbations, including long-range interactions, the diffusion constant can be expressed entirely in terms of generalized hydrodynamic data.
\end{abstract}

Hydrodynamics describes how many-body systems evolve from local to global equilibrium [1]. It can be regarded as an effective field theory for the transport of conserved quantities or other slow modes, assuming that all other modes relax parametrically faster. Hydrodynamics applies in a variety of contexts [2], from traditional classical fluid dynamics to quark-gluon plasmas [3, 4], black hole physics [5, 6], and electron fluids in graphene and $\mathrm{PdCoO}_{2}[7-9]$.

In one dimension, many paradigmatic models of quantum many-body physics - such as the Hubbard, Heisenberg, and Lieb-Liniger models - are integrable [10-22]. These models approximately describe experiments in quasi-one-dimensional materials and ultracold atomic gases [23-32]. Thus, approximate integrability is of wide experimental relevance. In nearly integrable systems, the short-time dynamics are integrable, feature infinitely many conservation laws, and are described by the recently developed framework of generalized hydrodynamics (GHD) [33-51]; at sufficiently long times, however, the dynamics are chaotic, feature finitely many conservation laws, and are typically described by conventional hydrodynamics. In integrable systems, transport is generically ballistic [36], although there are various limits that exhibit more exotic behavior [52-63]; in conventional hydrodynamics, one expects diffusion, unless the system possesses Galilean or Lorentz invariance [64-70]. The timescales governing the crossover between these two regimes have recently been explored both experimentally and numerically [28, 71], and have been shown to match a Fermi Golden Rule (FGR) prediction, with matrix elements evaluated via exact numerical diagonalization on small systems. However, except in noninteracting and weakly interacting models [72-77], the nature of relaxation and the transport coefficients governing the long-time hydrodynamics have not been investigated (see, e.g., Refs. 54, 78-81 for recent numerical studies). The existing perturbative results do not apply to many of the experimentally relevant settings, such as the Heisenberg and Fermi-Hubbard model, which are, in general, strongly interacting. Moreover, recent results suggest that anomalous transport might survive integrability breaking up to long times [59, 82-84], and it is crucial to construct a framework that captures how anomalous transport features due to integrability cross over to ordinary diffusion at long times.

In this work we develop a framework for computing relaxation and diffusion in nearly integrable systems, building on GHD. A central result of this work is a compact formula for the diffusion constant in nearly integrable systems with one (or a few) residual conservation laws. The specifics of the integrability-breaking mechanism enter this formula through a set of microscopic rates that govern the decay of the approximately conserved quantities. In general, these rates depend on the microscopic mechanism of integrability breaking. However, for integrability-breaking perturbations that are spatially slowly varying (e.g., smooth potentials and long-range interactions), these rates can themselves be expressed in terms of GHD data - in these cases, the diffusion constant can be fully expressed in terms of GHD data. Having introduced these general results using both the Kubo formula and a gradient expansion of the hydrodynamic equations, we apply them to some specific systems in which the physics is particularly transparent.

GHD Boltzmann equation.-Integrable systems have extensively many conserved quantities and stable, ballistically propagating quasiparticles, unlike quantum chaotic systems. Within GHD [33, 34], dynamics can be captured by a "Bethe-Boltzmann" equation for the density of quasiparticles, $\rho_{\lambda}(x, t)$, with a given quantum number (rapidity), $\lambda$ :

$$
\partial_{t} \rho_{\lambda}+\partial_{x}\left(v_{\lambda}^{\mathrm{eff}}[\rho] \rho_{\lambda}\right)=\mathcal{I}_{\lambda}[\rho] .
$$

where the effective group velocity $v_{\lambda}^{\text {eff }}[\rho]$ of quasiparticle type $\lambda$ is a functional of the densities of all quasiparticle types. The effective velocity can be computed from the thermodynamic Bethe ansatz (TBA) solution for the lo- 
cal state of the model, $\rho_{\lambda}(x, t)[33,34,85,86]$. Note that the quantum numbers $\lambda$ may be either discrete or continuous. Intuitively, Eq. (1) with a vanishing righthand side $\mathcal{I}_{\lambda}[\rho]=0$ is a kinetic equation that describes the ballistic propagation of the quasiparticles (solitons), which scatter elastically and delay one another through Wigner time delays [38, 41], leading to a state-dependent velocity $v_{\lambda}^{\text {eff }}[\rho]$. Since scattering processes in integrable systems factorize, this kinetic equation remains valid even if the quasiparticle gas is not dilute.

Breaking integrability endows this equation with a righthand side, $\mathcal{I}_{\lambda}[\rho]$, which accounts for the scrambling of the quasiparticle quantum numbers (see also Ref. 77). For simplicity, we restrict our consideration to fluctuations above homogeneous background states, $\rho(x, t)=$ $\rho^{\star}+\delta \rho(x, t)$. Since the dynamics of interest are not strictly integrable, the steady state, $\rho^{\star}$, will, in general, describe a standard thermal Gibbs ensemble. By definition, the righthand side of the Boltzmann equation vanishes for $\rho=\rho^{\star}$, so one can write $\mathcal{I}_{\lambda}[\rho]=$ $-\int d \theta \Gamma_{\lambda, \theta} \delta \rho_{\theta}(x, t)$, where $\Gamma_{\lambda, \theta} \equiv-\delta \mathcal{I}_{\lambda} /\left.\delta \rho_{\theta}\right|_{\rho=\rho^{\star}}$. The linearized version of Eq. (1) then reads

$$
\partial_{t} \delta \rho_{\lambda}+\partial_{x}(\mathbf{A} \delta \rho)_{\lambda}=-(\boldsymbol{\Gamma} \delta \rho)_{\lambda},
$$

where $\mathbf{A}$ and $\boldsymbol{\Gamma}$ are operators acting in rapidity space, e.g., as $(\boldsymbol{\Gamma} \delta \rho)_{\lambda}=\int d \theta \Gamma_{\lambda, \theta} \delta \rho_{\theta}(x, t)$. The matrix $\mathbf{A}$ is position independent: its expression in terms of the background state, $\rho^{\star}$, is known exactly in GHD [39] and its eigenvalues are the effective velocities $v_{\lambda}^{\text {eff }}\left[\rho^{\star}\right]$; the corresponding eigenvectors are the normal modes of GHD.

The densities of conserved quantities are given in terms of the quasiparticles by

$$
q_{m}(x, t)=\int d \lambda h_{m}(\lambda) \rho_{\lambda}(x, t),
$$

where $h$ is the charge carried by a quasiparticle with rapidity $\lambda$. The expectation value of the conserved charge $\hat{Q}_{m}$ in the generalized Gibbs ensemble (GGE) [21] corresponding to the background state, $\rho^{\star}$, is then given by $Q_{m} \equiv\left\langle\hat{Q}_{m}\right\rangle=\int d x q_{m}(x)$. In the charge basis, the deviation of the conserved charges from their background values follows from Eq. (2): $\partial_{t} \delta q_{n}+A_{n m} \partial_{x} \delta q_{m}=-\Gamma_{n m} \delta q_{m}$. Henceforth, repeated indices are implicitly summed over, and $\mathbf{A}$ and $\boldsymbol{\Gamma}$ are now written in the (complete) charge basis [87]. One has $A_{n m}=\int d \lambda d \theta h_{n}(\lambda) A_{\lambda, \theta} h_{m}(\theta)$ (and similarly for $\Gamma_{n m}$ ). Integrating this equation over position, $x$, one finds for $\boldsymbol{\Gamma} \neq 0$ that the charges decay as

$$
\delta \dot{Q}_{m}=-\Gamma_{m n} \delta Q_{n} .
$$

The eigenvalues of $\boldsymbol{\Gamma}$ give the decay rates of the quantities $\left\{Q_{m}\right\}$ that are conserved when $\boldsymbol{\Gamma}=0$; for $\boldsymbol{\Gamma} \neq 0$, the (decaying) eigenmodes of $\boldsymbol{\Gamma}$ are linear combinations of these $\left\{Q_{m}\right\}$ [88]. Any residual conserved quantities in the nonintegrable system, e.g., energy or particle number, correspond to zero modes of $\boldsymbol{\Gamma}$. In what follows, Greek characters denote residual conserved charges and Roman characters denote charges that decay when integrability is broken.

Kubo formula.-We now compute the linear response d.c. conductivity tensor, $\sigma_{\alpha \beta}$, of the residual conserved charges using the Kubo formula

$$
\sigma_{\alpha \beta}=\frac{1}{L} \int_{0}^{\infty} d t\left\langle\hat{J}_{\alpha}(t) \hat{J}_{\beta}(0)\right\rangle,
$$

evaluated in the GGE corresponding to the background state, $\rho^{\star}$, where $L$ is the system size and $\hat{J}_{\alpha}=\int d x \hat{j}_{\alpha}$ is the global current associated with the conserved charge $\hat{Q}_{\alpha}$. In the integrable limit $(\boldsymbol{\Gamma}=0)$, one can write $J_{\alpha}=J_{\alpha}^{\text {Euler }}+J_{\alpha}^{\text {fast }}$. When $\boldsymbol{\Gamma}=0$, the first term never decays because it can be decomposed onto conserved charges, $J_{\alpha}^{\text {Euler }}=A_{\alpha n} Q_{n}$, where $A_{n m}=\partial J_{n} / \partial Q_{m}$ are the components of $\mathbf{A}$ evaluated in the steady state, $\rho^{\star}$. The remaining fast components of the current generically relax on some characteristic timescale and give rise to diffusive and higher-order corrections to ballistic transport $[46-48,56]$.

On timescales at which the fast components have relaxed, one can take $J_{\alpha} \simeq A_{\alpha n} Q_{n}$ in Eq. (5) [89]. This yields $\left\langle\hat{J}_{\alpha}(t) \hat{J}_{\beta}(0)\right\rangle / L=A_{\alpha n} A_{\beta m} C_{n m}+\ldots$ at long times, where the matrix elements $C_{n m}=\left\langle\delta \hat{Q}_{n} \delta \hat{Q}_{m}\right\rangle / L$ encode the equilibrium fluctuations of the conserved charges [90], and are known exactly from TBA [91]. Thus, when $\boldsymbol{\Gamma}=0$, integrable dynamics generically lead to a nonzero value of the Kubo correlator and a Drude weight $D_{\alpha \beta}=$ $\lim _{t \rightarrow \infty}\left\langle\hat{J}_{\alpha}(t) \hat{J}_{\beta}(0)\right\rangle / L=\left(\mathbf{A C A}^{T}\right)_{\alpha \beta}$ [39]. This ballistic contribution to transport follows naturally from the overlap between currents and conserved charges, which prevents the currents from decaying at long times.

Conductivity tensor. - When $\boldsymbol{\Gamma} \neq 0$ all but a few charges decay according to Eq. (4), and one expects the currents to relax fully, giving rise to diffusive hydrodynamics. We assume that the currents are not modified by the integrability-breaking perturbation, which is justified perturbatively. The autocorrelator in Eq. (5) then relaxes in two stages: the fast component relaxes on a timescale of order unity and the Euler-scale component decays on a much longer timescale set by $\boldsymbol{\Gamma}$ (rather than persist indefinitely). At long times, one can ignore the contributions from the fast part (as before), which is subleading in $\boldsymbol{\Gamma}$, and expand the currents in terms of the slowly relaxing charges, $\delta Q_{n}(t)=[\exp (-\boldsymbol{\Gamma} t)]_{n m} \delta Q_{m}(0)$ to recover $\left\langle\hat{J}_{\alpha}(t) \hat{J}_{\beta}(0)\right\rangle / L=A_{\alpha n} A_{\beta m}\left[\mathrm{e}^{-\Gamma t}\right]_{n k} C_{k m}+\ldots$, where neglected terms include nonhydrodynamic modes that relax at a rate much faster than $\boldsymbol{\Gamma}$. Using the Kubo formula, Eq. (5), gives the d.c. conductivity tensor

$$
\sigma_{\alpha \beta}=\left(\mathbf{A} \boldsymbol{\Gamma}^{-1} \mathbf{A}\right)_{\alpha \gamma} C_{\gamma \beta},
$$

where unlabeled matrix products may be evaluated in either the charge or quasiparticle basis, but are restricted 
to the decaying modes - this projection onto nonconserved charges ensures that the inverse $\Gamma^{-1}$ is well defined. Thus, $\sigma_{\alpha \beta}$ is nonsingular and the d.c. limit is well-defined unless a current, $J_{\alpha}$, of a residual conserved charge, $Q_{\alpha}$, itself has some overlap with residual conserved charges, in which case $q_{\alpha}$ will spread ballistically even when integrability is broken. We also used $\mathbf{A C}=\mathbf{C A}^{T}$ [92], and that $C_{\alpha n}=0$ between quantities that are residually conserved for $\boldsymbol{\Gamma} \neq 0$ and those that are not, as the latter decay to zero. This means that the decaying and conserved charges belong to orthogonal subspaces under the hydrodynamic inner product defined by the matrix C. (If they did not, one could use this nonzero overlap to prove a Mazur bound, contradicting the assumption that these charges indeed decay [93].)

Eq. (6) is a central result of this work: it expresses the conductivity tensor entirely in terms of GHD data and the rate matrix, $\boldsymbol{\Gamma}$, governing the decay of the $\left\{Q_{m}\right\}$. Intuitively, this describes a generalized Drude formula: in the presence of integrability-breaking perturbations, the Drude weight for $\boldsymbol{\Gamma}=0$ is broadened into Lorentzians of width $\sim\|\boldsymbol{\Gamma}\|$ in the a.c. conductivity. Importantly, $\mathbf{A}$ and $\mathbf{C}$ are known exactly for integrable systems, and we will discuss below how $\boldsymbol{\Gamma}$ can be obtained in some cases from GHD data. Note that $\boldsymbol{\Gamma}$ can be efficiently inverted numerically - such kernel operator inversions are routinely performed in the solution of TBA equations.

We remark that diffusive corrections to ballistic transport in the integrable limit $(\boldsymbol{\Gamma}=0)$ are negligible compared to Eq. (6) in the limit where the integrabilitybreaking perturbation is small, unless the integrable model itself exhibits superdiffusion or diffusion if the Drude weight happens to vanish [36]. We will briefly return to this case below. Finally, if the spectrum of $\boldsymbol{\Gamma}$ is gapless (i.e., if it has eigenvalues arbitrarily close to zero) then ballistic transport may result in anomalous diffusion upon breaking integrability.

Diffusive hydrodynamics. - As the matrix $\mathbf{C}$ is also a susceptibility matrix, one can use a generalized Einstein relation to extract the diffusion matrix from Eq. (6),

$$
\mathcal{D}_{\alpha \beta}=\left(\mathbf{A} \boldsymbol{\Gamma}^{-1} \mathbf{A}\right)_{\alpha \beta},
$$

which will depend on the Lagrange multipliers, $\left\{\beta_{\gamma}\right\}$, of the GGE corresponding to the charges preserved by the integrability-breaking perturbation. Although Eq. (7) derives from linear response, $\mathcal{D}_{\alpha \beta}$ can be used as a transport coefficient to formulate a fully nonlinear hydrodynamic equation describing the dynamics at late times $t \gg \tau \sim\left\|\boldsymbol{\Gamma}^{-1}\right\|$,

$$
\partial_{t} \delta q_{\alpha}=\partial_{x}\left(\mathcal{D}_{\alpha \beta}\left[\left\{q_{\gamma}\right\}\right] \partial_{x} \delta q_{\beta}\right),
$$

where the Lagrange multipliers, $\left\{\beta_{\gamma}\right\}$, have been replaced by the expectation values of the conserved charges. (In principle Eq. (8) also includes a noise term, not shown, whose strength is fixed by the fluctuation-dissipation theorem.)
A more direct way to derive this diffusion equation in the linear response regime is as follows. For concreteness, we consider the case with a single residual conserved charge, $q_{0}$. The Euler-scale hydrodynamic equations are

$$
\begin{aligned}
\partial_{t} \delta q_{0}+A_{0 n} \partial_{x} \delta q_{n} & =0, \\
\partial_{t} \delta q_{n}+A_{n m} \partial_{x} \delta q_{m} & =-\Gamma_{n m} \delta q_{m}, \quad n \neq 0 .
\end{aligned}
$$

To leading order in the gradient expansion, we may drop derivatives of the $\delta q_{n}, \forall n \neq 0$ in the second equation, yielding $\delta q_{n}=-\Gamma_{n m}^{-1} A_{m 0} \partial_{x} \delta q_{0}+\ldots$ Substituting this into the first equation recovers the diffusion constant $\mathcal{D}_{00}=A_{0 n} \Gamma_{n m}^{-1} A_{m 0}$, consistent with the Kubo result, Eq. (7) [94]. We emphasize that here diffusion arises from "integrating out" slow but nonconserved degrees of freedom, and is dramatically different from the diffusive corrections that arise in integrable systems $(\boldsymbol{\Gamma}=$ 0 ) due to the fluctuations of ballistically propagating modes [47, 95, 96].

Note that the preceding arguments do not rely on spatial locality of $\boldsymbol{\Gamma}$, and, in fact, generalize to the case wherein $\boldsymbol{\Gamma}$ is a spatially nonlocal kernel. In that case, the diffusion equation takes the form $\partial_{t} q_{0}(x, t)=\partial_{x}\left\{\int d y \mathcal{D}(x-y) \partial_{y} q_{0}(y)\right\}$, where $\mathcal{D}(x-y) \equiv$ $A_{0 n}(x) \Gamma_{n m}^{-1}(x-y) A_{m 0}(y)$. When $\mathcal{D}(x-y)$ is sufficiently long ranged, the nature of the hydrodynamics might change, though we will not consider this case in detail.

Hydrodynamic projections and general operators.- - In the discussion above, we analyzed the dynamics of current autocorrelators. However, the essential ingredientnamely, the separation of an operator into fast and slow components, where the latter correspond to overlaps of the operator with almost conserved charges - is true for any operator. Thus, the analysis above directly generalizes to the autocorrelation function of an arbitrary "global" operator $\hat{\mathcal{O}}=\sum_{i} \hat{\mathcal{O}}_{i}$, via the formalism of hydrodynamic projections (see, e.g., Ref. 97). For simplicity, we assume that $\langle\hat{\mathcal{O}}\rangle=0$ in the GGE associated with the background state, $\rho^{\star}$. The projection of $\hat{\mathcal{O}}$ onto a slow (but nonconserved) charge, $Q_{m}$ can be expressed as

$$
\left\langle\mathcal{O} \mid Q_{m}\right\rangle=-\left.\partial_{\beta_{m}}\langle\hat{\mathcal{O}}\rangle\right|_{\beta_{m}=0},
$$

When $\boldsymbol{\Gamma}=0$, one can use the TBA formalism to compute expectation values for any value of the chemical potential, $\beta_{m}$, associated with the charge $Q_{m}$, and can thus evaluate the projection for sufficiently simple operators. It readily follows that

$$
\langle\hat{\mathcal{O}}(t) \hat{\mathcal{O}}(0)\rangle=\left\langle\mathcal{O} \mid Q_{m}\right\rangle C_{m n}^{-1}\left[e^{-\Gamma t}\right]_{n p}\left\langle Q_{p} \mid \mathcal{O}\right\rangle .
$$

For current operators $J_{\alpha}$, the hydrodynamic projection $\left\langle J_{\alpha} \mid Q_{n}\right\rangle=B_{\alpha n}=-\partial J_{\alpha} / \partial \beta_{n}$ defines the matrix $\mathbf{B}=$ AC (by the chain rule) [92], which recovers Eq. (6).

Transition rates. - So far, we have expressed the behavior of autocorrelation functions in terms of GHD data and the matrix $\boldsymbol{\Gamma}$, which describes the decay of the conserved 
charges due to collisions. We now discuss how one can compute $\boldsymbol{\Gamma}$ perturbatively. From Fermi's Golden Rule, the RHS of Eq. (1) takes the general schematic form

$$
\begin{array}{r}
\mathcal{I}_{\lambda}=\int \prod_{i j} d \alpha_{i} d \beta_{j}\left(\prod_{i j} \rho_{\alpha_{i}} \rho_{\beta_{j}}^{h} \rho_{\lambda}^{h}\left|M_{\left\{\alpha_{i}\right\} \rightarrow\left\{\beta_{j}, \lambda\right\}}\right|^{2}\right. \\
\left.-\rho_{\lambda} \prod_{i j} \rho_{\beta_{j}} \rho_{\alpha_{i}}^{h}\left|M_{\left\{\lambda, \beta_{j}\right\} \rightarrow\left\{\alpha_{i}\right\}}\right|^{2}\right)
\end{array}
$$

where $\rho_{\alpha}^{h}$ is the density of holes with rapidity $\alpha$. We also introduce the density of states, $\rho_{\lambda}^{\text {tot }}=\rho_{\lambda}+\rho_{\lambda}^{h}$, and the occupation factor, $n_{\lambda}=\rho_{\lambda} / \rho_{\lambda}^{\text {tot }}$. Here, $M$ denotes matrix elements of the integrability-breaking perturbation between eigenstates of the integrable system. The first term corresponds to scattering particles into the quasiparticle state $\lambda$, and the second to scattering them out. The scattering can happen in various permutations, which must be summed over. In general, the matrix elements that enter this expression must be derived from microscopics; however, in some cases, they can be expressed in terms of GHD data in the hydrodynamic limit.

As a simple example we consider an interacting onedimensional Bose gas (the Lieb-Liniger model with particle mass $m$ ) subject to a weak, smoothly varying timedependent potential coupled to one of the charges [51], i.e. $V(x) \eta(t) \hat{\rho}(x)$, with $\hat{\rho}=\hat{q}_{0}$, the quasiparticle density. (For the less trivial case of an interaction with a smooth kernel see [94].) A key observation [48, 98] is that, at long wavelengths, the dominant matrix elements of $V(x) \hat{\rho}(x)$ are those that rearrange the fewest quasiparticles, regardless of interaction strength. Thus we can restrict to oneparticle-hole excitations, for which the matrix elements are given by $\left\langle m\left|\hat{q}_{0}\right| m ;\{\lambda \rightarrow \theta\}\right\rangle=h_{0}^{\mathrm{dr}}(\lambda)[48]$, with $h_{0}^{\mathrm{dr}}$ the "dressed" charge, $h_{0}^{\mathrm{dr}}=(\mathbf{1}+\mathbf{n K})^{-1} h_{0}$ where $h_{0}(\lambda)=1$ for particle number, $\mathbf{K}$ is the scattering kernel of the model, and $\mathbf{n}$ acts diagonally in rapidity space as the occupation factor, $n_{\lambda}$. We find that

$$
\begin{aligned}
\mathcal{I}_{\lambda}= & \rho_{\lambda}^{\mathrm{tot}} \int d \varphi\left|\tilde{V}\left(k_{\lambda+\varphi}-k_{\lambda}\right)\right|^{2}\left|\tilde{\eta}\left(\varepsilon_{\lambda+\varphi}-\varepsilon_{\lambda}\right)\right|^{2} \\
& \rho_{\lambda+\varphi}^{\mathrm{tot}} h_{0}^{\mathrm{dr}}(\lambda) h_{0}^{\mathrm{dr}}(\lambda+\varphi)\left[n_{\lambda+\varphi}\left(1-n_{\lambda}\right)-n_{\lambda}\left(1-n_{\lambda+\varphi}\right)\right]
\end{aligned}
$$

where $\tilde{V}, \tilde{\eta}$ denote Fourier transforms and $\varepsilon_{\lambda}$ and $k_{\lambda}$ are, respectively, the dressed energy and momentum of the excitation, satisfying $\varepsilon^{\prime}=\left(E^{\prime}\right)^{\mathrm{dr}}$ and $k^{\prime}=\left(P^{\prime}\right)^{\mathrm{dr}}$, with $E(\lambda)=m \lambda^{2} / 2$ and $P(\lambda)=m \lambda$ the single-particle energy and momentum (entering Eq. (3)). If we fix the background state, this is similar to the scattering of free fermions with charge $h_{0}^{\mathrm{dr}}$. This dependence on the dressed charge is also seen in the more complicated case of slowly varying interactions [94]. However, our assumptions fail for many important types of scatterings within an integrable system, such as the decay of one quasiparticle type into another or Umklapp scattering of quasiparticles; incorporating these is an interesting topic for future work.
Examples.-We now comment on the physical significance of our results by considering several specific cases. First, consider an interacting Bose gas in one dimension with particle mass $m$. By Galilean invariance the current corresponding to the boson density, $q_{0}$, is the momentum, $j_{0}=q_{1}$. Suppose that the momentum distribution relaxes to a Gaussian on a timescale $\tau$ (e.g., due to point scatterers). Then Eq. (6) predicts a conductivity $\sigma=\tau \chi A_{01} A_{10}$ for the boson density. We have $A_{01}=1$ by Galilean invariance, and $A_{10}=\chi^{-1} n / m$ (see e.g. [99]). We thus recover the Drude formula $\sigma=\tau n / m$ (momentum relaxation time times Drude weight). A similar result would apply to energy transport in the spin-1/2 XXZ chain with pointlike scatterers.

Next, we turn to cases in which corrections to the Euler scale are large and potentially even divergent. An example is the spin-1/2 XXZ chain with easy-plane anisotropy. For concreteness, we consider subjecting this system to slowly varying noise, as before. In this model, the Drude weight varies discontinuously with the anisotropy parameter [36, 44, 100-104], and, concomitantly, the low-frequency response is anomalous, so that in the integrable limit, for generic anisotropy, one has $\sigma(\omega)=D \delta(\omega)+c \omega^{-1 / 2}[62]$. Only the largest quasiparticles ("strings") in this model are charged under the magnetization, so only these strings couple to the integrability-breaking perturbation. In the integrable limit, these strings undergo a Lévy flight; when integrability is broken, the Lévy flight is cut off and crosses over to diffusion with a mean free time $\tau$. The d.c. conductivity then goes as $\sigma=D \tau+c \sqrt{\tau}$, i.e., it corresponds to convolving the integrable result with a Lorentzian of width $\tau^{-1}$. Note that this result is nonanalytic in $\tau$ : anomalous transport in the integrable limit can result in signatures in the nonanalytic dependence of the diffusion constant on the integrability-breaking parameter. One can try to extend this analysis to the easy-axis regime of the XXZ model where spin transport is diffusive in the integrable limit; in this regime, none of the quasiparticles carry any dressed magnetization, thus at the present level of analysis their relaxation rates vanish. However, spin transport remains diffusive upon breaking integrability: the spin Drude weight is zero since the quasiparticles are neutral, but the factors of dressed magnetization cancel out in eq. (7), so we predict a finite diffusion constant which can be computed by adding a small magnetic field $h$ (which makes the Drude weight and relaxation rates non-zero) and taking $h \rightarrow 0$ in (7).

Conclusion.-This work has shown how the crossover from generalized to conventional hydrodynamics can be captured within the framework of GHD, by introducing a collision integral into the Bethe-Boltzmann equation. GHD allows one to write compact formulas for the diffusion constants of the residual conserved quantities, as well as for more general autocorrelation functions; it also gives access to the full, potentially nonlinear and spa- 
tially nonlocal, diffusion equations for the residual conserved quantities. These formulas involve hydrodynamic data as well as a matrix of quasiparticle decay rates, which (in the most general case) lies beyond the scope of GHD. Nevertheless, in certain limits where the collisions involve small momentum transfer, the rates can themselves be expressed in terms of GHD data, thus allowing for a fully GHD description of nearly integrable systems. Applying this technology to extract specific quantitative predictions for experiments, by incorporating the collision integral into the flea-gas algorithm [41] for integrable dynamics, is a natural avenue for future work [105].

Acknowledgments.-The authors thank Philipp Dumitrescu, Vadim Oganesyan, and especially Vir Bulchandani for useful discussions. This work was supported by the National Science Foundation under NSF Grant No. DMR-1653271 (S.G.), the US Department of Energy, Office of Science, Basic Energy Sciences, under Early Career Award No. DE-SC0019168 (R.V.), and the Alfred P. Sloan Foundation through a Sloan Research Fellowship (R.V.).

[1] L. D. Landau and E. M. Lifshitz, Course of theoretical physics. vol. 6: Fluid mechanics (Oxford, 1987).

[2] P. Glorioso and H. Liu, arXiv e-prints , arXiv:1805.09331 (2018), arXiv:1805.09331 [hep-th].

[3] D. A. Teaney, "Viscous Hydrodynamics and the Quark Gluon Plasma," in Quark-Gluon Plasma (2010) pp. 207266.

[4] P. Romatschke and U. Romatschke, arXiv e-prints , arXiv:1712.05815 (2017), arXiv:1712.05815 [nucl-th].

[5] D. T. Son and A. O. Starinets, Annual Review of Nuclear and Particle Science 57, 95 (2007), https://doi.org/10.1146/annurev.nucl.57.090506.123120.

[6] V. E. Hubeny, S. Minwalla, and M. Rangamani, arXiv e-prints , arXiv:1107.5780 (2011), arXiv:1107.5780 [hepth].

[7] D. A. Bandurin, I. Torre, R. K. Kumar, M. Ben Shalom, A. Tomadin, A. Principi, G. H. Auton, E. Khestanova, K. S. Novoselov, I. V. Grigorieva, L. A. Ponomarenko, A. K. Geim, and M. Polini, Science 351, 1055 (2016).

[8] J. Crossno, J. K. Shi, K. Wang, X. Liu, A. Harzheim, A. Lucas, S. Sachdev, P. Kim, T. Taniguchi, K. Watanabe, T. A. Ohki, and K. C. Fong, Science 351, 1058 (2016).

[9] P. J. W. Moll, P. Kushwaha, N. Nandi, B. Schmidt, and A. P. Mackenzie, Science 351, 1061 (2016).

[10] P. Calabrese and J. Cardy, Physical Review Letters 96, 136801 (2006).

[11] J. Sirker, R. G. Pereira, and I. Affleck, Phys. Rev. Lett. 103, 216602 (2009).

[12] T. Prosen, Phys. Rev. Lett. 106, 217206 (2011).

[13] J.-S. Caux and F. H. L. Essler, Phys. Rev. Lett. 110, 257203 (2013).

[14] B. Wouters, J. De Nardis, M. Brockmann, D. Fioretto, M. Rigol, and J.-S. Caux, Phys. Rev. Lett. 113, 117202 (2014).
[15] E. Ilievski, J. De Nardis, B. Wouters, J.-S. Caux, F. H. L. Essler, and T. Prosen, Phys. Rev. Lett. 115, 157201 (2015).

[16] B. Pozsgay, M. Mestyán, M. A. Werner, M. Kormos, G. Zaránd, and G. Takács, Phys. Rev. Lett. 113, 117203 (2014).

[17] E. Ilievski, M. Medenjak, T. Prosen, and L. Zadnik, Journal of Statistical Mechanics: Theory and Experiment 2016, 064008 (2016).

[18] P. Calabrese, F. H. L. Essler, and G. Mussardo, Journal of Statistical Mechanics: Theory and Experiment 2016, 064001 (2016).

[19] F. H. L. Essler and M. Fagotti, Journal of Statistical Mechanics: Theory and Experiment 2016, 064002 (2016).

[20] R. Vasseur and J. E. Moore, Journal of Statistical Mechanics: Theory and Experiment 2016, 064010 (2016).

[21] L. Vidmar and M. Rigol, Journal of Statistical Mechanics: Theory and Experiment 2016, 064007 (2016).

[22] M. Fagotti, M. Collura, F. H. L. Essler, and P. Calabrese, Phys. Rev. B 89, 125101 (2014).

[23] T. Kinoshita, T. Wenger, and D. Weiss, Nature 440, 900 (2006).

[24] M. Gring, M. Kuhnert, T. Langen, T. Kitagawa, B. Rauer, M. Schreitl, I. Mazets, D. A. Smith, E. Demler, and J. Schmiedmayer, Science 337, 1318 (2012).

[25] J. P. Ronzheimer, M. Schreiber, S. Braun, S. S. Hodgman, S. Langer, I. P. McCulloch, F. Heidrich-Meisner, I. Bloch, and U. Schneider, Phys. Rev. Lett. 110, 205301 (2013).

[26] L. Vidmar, J. P. Ronzheimer, M. Schreiber, S. Braun, S. S. Hodgman, S. Langer, F. Heidrich-Meisner, I. Bloch, and U. Schneider, Phys. Rev. Lett. 115, 175301 (2015).

[27] S. Scherg, T. Kohlert, J. Herbrych, J. Stolpp, P. Bordia, U. Schneider, F. Heidrich-Meisner, I. Bloch, and M. Aidelsburger, Phys. Rev. Lett. 121, 130402 (2018).

[28] Y. Tang, W. Kao, K.-Y. Li, S. Seo, K. Mallayya, M. Rigol, S. Gopalakrishnan, and B. L. Lev, Phys. Rev. X 8, 021030 (2018).

[29] S. Erne, R. Bücker, T. Gasenzer, J. Berges, and J. Schmiedmayer, Nature 563, 225 (2018).

[30] L. A. Zundel, J. M. Wilson, N. Malvania, L. Xia, J.-F. Riou, and D. S. Weiss, Phys. Rev. Lett. 122, 013402 (2019).

[31] M. Schemmer, I. Bouchoule, B. Doyon, and J. Dubail, Phys. Rev. Lett. 122, 090601 (2019).

[32] C. Hess, Physics Reports 811, 1 (2019).

[33] O. A. Castro-Alvaredo, B. Doyon, and T. Yoshimura, Phys. Rev. X 6, 041065 (2016).

[34] B. Bertini, M. Collura, J. De Nardis, and M. Fagotti, Phys. Rev. Lett. 117, 207201 (2016).

[35] B. Doyon and T. Yoshimura, SciPost Phys. 2, 014 (2017).

[36] E. Ilievski and J. De Nardis, Phys. Rev. Lett. 119, 020602 (2017).

[37] V. B. Bulchandani, R. Vasseur, C. Karrasch, and J. E. Moore, Phys. Rev. Lett. 119, 220604 (2017).

[38] V. B. Bulchandani, R. Vasseur, C. Karrasch, and J. E. Moore, Phys. Rev. B 97, 045407 (2018).

[39] B. Doyon and H. Spohn, SciPost Phys. 3, 039 (2017).

[40] B. Doyon and H. Spohn, Journal of Statistical Mechanics: Theory and Experiment 2017, 073210 (2017).

[41] B. Doyon, T. Yoshimura, and J.-S. Caux, Phys. Rev. Lett. 120, 045301 (2018).

[42] B. Doyon, J. Dubail, R. Konik, and T. Yoshimura, Phys. Rev. Lett. 119, 195301 (2017). 
[43] X. Zotos, ArXiv e-prints (2016), arXiv:1604.08434 [condmat.stat-mech].

[44] E. Ilievski and J. De Nardis, Phys. Rev. B 96, 081118 (2017).

[45] M. Collura, A. De Luca, and J. Viti, Phys. Rev. B 97, 081111 (2018).

[46] J. De Nardis, D. Bernard, and B. Doyon, Phys. Rev. Lett. 121, 160603 (2018).

[47] S. Gopalakrishnan, D. A. Huse, V. Khemani, and R. Vasseur, Phys. Rev. B 98, 220303 (2018).

[48] J. D. Nardis, D. Bernard, and B. Doyon, SciPost Phys. 6, 49 (2019).

[49] D. X. Horvath, arXiv preprint arXiv:1905.08590 (2019).

[50] B. Bertini, L. Piroli, and M. Kormos, Phys. Rev. B 100, 035108 (2019).

[51] A. Bastianello, V. Alba, and J.-S. Caux, Phys. Rev. Lett. 123, 130602 (2019).

[52] T. Prosen and M. Žnidarič, Journal of Statistical Mechanics: Theory and Experiment 2009, P02035 (2009).

[53] M. Ljubotina, M. Žnidarič, and T. Prosen, Nature Communications 8, 16117 EP (2017).

[54] R. J. Sánchez, V. K. Varma, and V. Oganesyan, Physical Review B 98, 054415 (2018).

[55] E. Ilievski, J. De Nardis, M. Medenjak, and T. Prosen, Phys. Rev. Lett. 121, 230602 (2018).

[56] S. Gopalakrishnan and R. Vasseur, Phys. Rev. Lett. 122, 127202 (2019).

[57] U. Agrawal, S. Gopalakrishnan, and R. Vasseur, Phys. Rev. B 99, 174203 (2019).

[58] S. Gopalakrishnan, R. Vasseur, and B. Ware, Proceedings of the National Academy of Sciences 116, 16250 (2019).

[59] J. De Nardis, M. Medenjak, C. Karrasch, and E. Ilievski, Phys. Rev. Lett. 123, 186601 (2019).

[60] M. Ljubotina, M. Žnidarič, and T. Prosen, Phys. Rev, Lett. 122, 210602 (2019).

[61] A. Das, M. Kulkarni, H. Spohn, and A. Dhar, arXiv preprint arXiv:1906.02760 (2019).

[62] U. Agrawal, S. Gopalakrishnan, R. Vasseur, and B. Ware, arXiv e-prints , arXiv:1909.05263 (2019), arXiv:1909.05263 [cond-mat.stat-mech].

[63] V. B. Bulchandani, arXiv e-prints , arXiv:1910.08266 (2019), arXiv:1910.08266 [cond-mat.stat-mech].

[64] H. van Beijeren, Phys. Rev. Lett. 108, 180601 (2012).

[65] M. Kulkarni and A. Lamacraft, Phys. Rev. A 88, 021603 (2013).

[66] H. Spohn, Journal of Statistical Physics 154, 1191 (2014).

[67] C. B. Mendl and H. Spohn, Phys. Rev. Lett. 111, 230601 (2013).

[68] M. Kulkarni, D. A. Huse, and H. Spohn, Phys. Rev. A 92, 043612 (2015).

[69] A. Miron, J. Cividini, A. Kundu, and D. Mukamel, Phys. Rev. E 99, 012124 (2019).

[70] A. Das, K. Damle, A. Dhar, D. A. Huse, M. Kulkarni, C. B. Mendl, and H. Spohn, Journal of Statistical Physics (2019), 10.1007/s10955-019-02397-y.

[71] K. Mallayya, M. Rigol, and W. De Roeck, Phys. Rev. X 9, 021027 (2019).

[72] L. Landau, E. Lifshitz, and L. Pitaevskij, Course of theoretical physics. vol. 10: Physical kinetics (Oxford, 1981).

[73] P. Jung, R. W. Helmes, and A. Rosch, Phys. Rev. Lett. 96, 067202 (2006).
[74] G. P. Brandino, J.-S. Caux, and R. M. Konik, Phys. Rev. X 5, 041043 (2015).

[75] B. Bertini, F. H. L. Essler, S. Groha, and N. J. Robinson, Phys. Rev. Lett. 115, 180601 (2015).

[76] B. Bertini, F. H. L. Essler, S. Groha, and N. J. Robinson, Phys. Rev. B 94, 245117 (2016).

[77] J.-S. Caux, B. Doyon, J. Dubail, R. Konik, and T. Yoshimura, SciPost Phys. 6, 70 (2019).

[78] L. F. Santos and M. Rigol, Phys. Rev. E 81, 036206 (2010).

[79] K. Mallayya and M. Rigol, Phys. Rev. Lett. 120, 070603 (2018).

[80] K. Mallayya and M. Rigol, Phys. Rev. Lett. 123, 240603 (2019).

[81] A. Biella, M. Collura, D. Rossini, A. De Luca, and L. Mazza, Nature Communications 10, 4820 (2019).

[82] X. Cao, V. B. Bulchandani, and J. E. Moore, Phys. Rev. Lett. 120, 164101 (2018).

[83] V. B. Bulchandani, C. Karrasch, and J. E. Moore, arXiv e-prints , arXiv:1904.09287 (2019), arXiv:1904.09287 [cond-mat.stat-mech].

[84] M. Dupont and J. E. Moore, arXiv preprint arXiv:1907.12115 (2019).

[85] L. Bonnes, F. H. L. Essler, and A. M. Läuchli, Phys. Rev. Lett. 113, 187203 (2014).

[86] M. Borsi, B. Pozsgay, and L. Pristyák, arXiv e-prints , arXiv:1908.07320 (2019), arXiv:1908.07320 [cond-mat.stat-mech].

[87] We are free to choose superpositions of the $Q_{m}$ for which the functions $h_{m}(\lambda)$ have a simple completeness relation.

[88] We require $\boldsymbol{\Gamma}$ to be positive semidefinite: its eigenvalues, $\{\gamma\}$ obey $\operatorname{Re}(\gamma) \geq 0$

[89] This Euler-scale relation is, strictly speaking, valid for the expectation values in the GGE, but is not an operator identity; hence the lack of hats.

[90] Because of the way conserved charges are defined, the matrix $\mathbf{C}$ is nontrivial even in the infinite-temperature thermal state.

[91] P. Fendley and H. Saleur, Phys. Rev. B 54, 10845 (1996).

[92] H. Spohn, Large scale dynamics of interacting particles, Texts and monographs in physics (Springer-Verlag, 1991).

[93] V. B. Bulchandani, private communication.

[94] See Supplemental Information for a systematic analysis of the hydrodynamics equations with integrabilitybreaking terms, and an analysis of the Golden-Rule rates for long-range interactions.

[95] M. Medenjak, J. De Nardis, and T. Yoshimura, arXiv e-prints , arXiv:1911.01995 (2019), arXiv:1911.01995 [cond-mat.stat-mech].

[96] B. Doyon, arXiv e-prints , arXiv:1912.01551 (2019), arXiv:1912.01551 [cond-mat.stat-mech].

[97] B. Doyon, SciPost Phys. 5, 54 (2018).

[98] J. De Nardis and M. Panfil, Journal of Statistical Mechanics: Theory and Experiment 2018, 033102 (2018).

[99] R. Vasseur, C. Karrasch, and J. E. Moore, Phys. Rev. Lett. 115, 267201 (2015).

[100] X. Zotos, Phys. Rev. Lett. 82, 1764 (1999).

[101] C. Karrasch, J. H. Bardarson, and J. E. Moore, Phys. Rev. Lett. 108, 227206 (2012).

[102] T. Prosen, Nuclear Physics B 886, 1177 (2014).

[103] T. Prosen and E. Ilievski, Phys. Rev. Lett. 111, 057203 (2013).

[104] A. Urichuk, Y. Oez, A. Klümper, and J. Sirker, SciPost 
Physics 6, 005 (2019).

[105] A. J. Friedman et al., in preparation. 


\title{
Supplemental Material for "Diffusive hydrodynamics from integrability breaking"
}

\author{
Aaron J. Friedman ${ }^{1,2}$, Sarang Gopalakrishnan ${ }^{3}$, and Romain Vasseur ${ }^{2}$ \\ ${ }^{1}$ Department of Physics and Astronomy, University of California, Irvine, CA 92697, USA \\ ${ }^{2}$ Department of Physics, University of Massachusetts, Amherst, Massachusetts 01003, USA \\ ${ }^{3}$ Department of Physics and Astronomy, CUNY College of Staten Island, \\ Staten Island, NY 10314; Physics Program and Initiative for the Theoretical Sciences, \\ The Graduate Center, CUNY, New York, NY 10016, USA
}

\section{SOLUTION OF THE LINEARIZED HYDRODYNAMIC EQUATIONS}

Our starting point are the hydrodynamic equations:

$$
\begin{aligned}
\partial_{t} \delta q_{0}+A_{0 n} \partial_{x} \delta q_{n} & =0, \\
\partial_{t} \delta q_{n}+A_{n m} \partial_{x} \delta q_{m} & =-\Gamma_{n p} \delta q_{p}, \quad n \neq 0 .
\end{aligned}
$$

Here, $Q_{0}$ is conserved by the integrability breaking perturbation, while all the other charges decay slowly at a rate set by $\boldsymbol{\Gamma}$. To solve the dynamics, we invert the second equation to get

$$
\delta q_{n}=-\left(\boldsymbol{\Gamma}^{-1} \partial_{t}+\boldsymbol{\Gamma}^{-1} \mathbf{A} \partial_{x}\right)_{n 0} \delta q_{0}-\sum_{m \neq 0}\left(\boldsymbol{\Gamma}^{-1} \partial_{t}+\boldsymbol{\Gamma}^{-1} \mathbf{A} \partial_{x}\right)_{n m} \delta q_{m},
$$

where all matrix products and inverses are within the non-conserved subspace $n \neq 0$. We can formally solve this equation by iterations, in the limit $\omega \tau \ll 1$ and $k \ell \ll 1$, with $\omega \sim \partial_{t}$ the frequency, $k \sim \partial_{x}$ the momentum, $\tau \sim\left\|\boldsymbol{\Gamma}^{-1}\right\|$ the typical relaxation time, and $\ell=v^{\mathrm{eff}} \tau$ the mean free path with $v^{\mathrm{eff}} \sim\|\mathbf{A}\|$ a velocity scale. This allows us to find a formal expression of the non-conserved charges in terms of $\delta q_{0}$

$$
\delta q_{n}=\sum_{m \geq 1}(-1)^{m}\left[\left(\boldsymbol{\Gamma}^{-1} \partial_{t}+\boldsymbol{\Gamma}^{-1} \mathbf{A} \partial_{x}\right)^{m}\right]_{n 0} \delta q_{0} \approx-\left(\boldsymbol{\Gamma}^{-1} \mathbf{A}\right)_{n 0} \partial_{x} \delta q_{0}+\ldots
$$

to leading order in the gradient expansion. Using this expression in the continuity equation for $\delta q_{0}(1)$, we find

$$
\partial_{t} \delta q_{0}=\left(\mathbf{A} \boldsymbol{\Gamma}^{-1} \mathbf{A}\right)_{00} \partial_{x}^{2} \delta q_{0}
$$

As expected, we find a diffusion equation for the charge $\delta q_{0}$, with a diffusion constant $\mathcal{D}=\left(\mathbf{A} \boldsymbol{\Gamma}^{-1} \mathbf{A}\right)_{00}$.

\section{GOLDEN-RULE RATES FOR SLOWLY VARYING INTERACTIONS}

We consider integrability-breaking interactions of the form $V(x-y) \hat{q}(x) \hat{q}(y)$. We assume that the interaction is asymptotically sufficiently short-range, i.e., $V(x-y) \lesssim 1 /|x-y|^{2}$ as $|x-y| \rightarrow \infty$; however, we also take it to be longrange enough that the associated momentum transfer is small. We also assume that most of the particles that interact are farther than a thermal correlation length away from one another, so that, between two many-body eigenstates, $\langle m|\hat{q}(x) \hat{q}(y)| n\rangle \sim\langle m|\hat{q}(x)| n\rangle \otimes\left\langle m^{\prime}|\hat{q}(y)| n^{\prime}\right\rangle$. In the latter expression, the quantum states are representative local eigenstates drawn from the appropriate Gibbs ensemble.

As we noted in the main text, in this long-wavelength limit, the dominant many-body matrix elements are those that involve rearranging as few particles as possible. (These are dominant in the sense that the one- and two-particle processes essentially exhaust the relevant sum rule [1].) In one dimension, elastic two-body collisions do not relax momentum or energy; thus the lowest-order usable processes are Umklapp scattering (which occurs only in lattice models, and requires large momentum transfer, thus falling outside the scope of our discussion) and diffractive threebody scattering (see [2]). We consider the latter process in the limit of small momentum transfer.

One can make up a three-body process by using either $\hat{q}$ to rearrange two quasiparticles and the other to rearrange a single quasiparticle. The matrix element of the density operator for rearranging a single quasiparticle of rapidity $\lambda$ is $h^{\mathrm{dr}}(\lambda)$, as noted in the main text. For the two-body process, the form of the matrix element in general is conjectured 
to take the general form [Ref. [3], Eq. (3.49), see also [4]]

$$
\begin{gathered}
\left\langle m|\hat{q}| m ;\left\{\theta_{1}^{h}, \theta_{2}^{h}\right\} \rightarrow\left\{\theta_{1}^{p}, \theta_{2}^{p}\right\}\right\rangle=2 \pi\left(\theta_{1}^{p}+\theta_{2}^{p}-\theta_{1}^{h}-\theta_{2}^{h}\right)\left(\frac{K_{\theta_{2}^{h}}^{\mathrm{dr}} \theta_{1}^{h} h^{\mathrm{dr}}\left(\theta_{2}^{h}\right)}{k_{\theta_{1}^{h}}^{\prime} k_{\theta_{2}^{h}}^{\prime}\left(\theta_{1}^{p}-\theta_{1}^{h}\right)}+\frac{K_{\theta_{1}^{h}, \theta_{2}^{h}}^{\mathrm{dr}} h^{\mathrm{dr}}\left(\theta_{1}^{h}\right)}{k_{\theta_{1}^{h}}^{\prime} k_{\theta_{2}^{h}}^{\prime}\left(\theta_{2}^{p}-\theta_{2}^{h}\right)}\right. \\
\left.+\frac{K_{\theta_{2}^{h}}^{\mathrm{dr}} \theta_{1}^{h} h^{\mathrm{dr}}\left(\theta_{2}^{h}\right)}{k_{\theta_{1}^{h}}^{\prime} k_{\theta_{2}^{h}}^{\prime}\left(\theta_{2}^{p}-\theta_{1}^{h}\right)}+\frac{K_{\theta_{1}^{h}}^{\mathrm{dr}}, \theta_{2}^{h} h^{\mathrm{dr}}\left(\theta_{1}^{h}\right)}{k_{\theta_{1}^{h}}^{\prime} k_{\theta_{2}^{h}}^{\prime}\left(\theta_{1}^{p}-\theta_{2}^{h}\right)}\right),
\end{gathered}
$$

with $\mathbf{K}^{\mathrm{dr}}=(\mathbf{1}+\mathbf{n K})^{-1} \mathbf{K}$ the dressed scattering kernel. Here, $\theta_{1,2}^{h}$ are arbitrary but close to $\theta_{1,2}^{p}$, i.e., this form is reliable close to the poles; for slowly varying interactions, however, the momentum transfer is small and the matrix element is large only in the vicinity of the poles, so Eq. (5) is a valid approximation. In terms of these matrix elements, the collision integral can be expressed as

$$
\begin{array}{rl}
\mathcal{I}_{\lambda}=\int d \theta_{0}^{h} & d \theta_{1}^{h} d \theta_{2}^{h} d \theta_{1}^{p} d \theta_{2}^{p}\left[\tilde{V}\left(k_{\theta_{0}^{h}}-k_{\lambda}\right)\right]^{2} \rho_{\theta_{0}^{h}} \rho_{\theta_{1}^{h}} \rho_{\theta_{2}^{h}} \rho_{\theta_{1}^{p}}^{h} \rho_{\theta_{2}^{p}}^{h} \rho_{\lambda}^{h} \\
\times & \left.\times(2 \pi)^{2} \delta\left[k_{\theta_{0}^{h}}+k_{\theta_{1}^{h}}+k_{\theta_{2}^{h}}-k_{\theta_{1}^{p}}-k_{\theta_{2}^{p}}-k_{\lambda}\right] \delta\left[\varepsilon_{\theta_{0}^{h}}+\varepsilon_{\theta_{1}^{h}}+\varepsilon_{\theta_{2}^{h}}-\varepsilon_{\theta_{1}^{p}}-\varepsilon_{\theta_{2}^{p}}-\varepsilon_{\lambda}\right)\right] \\
\times & {\left[h^{\mathrm{dr}}\left(\theta_{0}^{h}\right)\right]^{2}\left|\left\langle m|\hat{q}| m ;\left\{\theta_{1}^{h}, \theta_{2}^{h}\right\} \rightarrow\left\{\theta_{1}^{p}, \theta_{2}^{p}\right\}\right\rangle\right|^{2}+\text { permutations. }}
\end{array}
$$

Here, we have only shown the first in-scattering term, in which a quasiparticle is scattered into the state $\lambda$ by a three-body collision; its permutations include two other in-scattering terms, in which one of the other final states is $\lambda$, as well as three out-scattering terms in which one of the $\theta^{h}$ is replaced by $\lambda$. Eqs. (6) and (5) together specify the collision integral purely in terms of GHD data, albeit in a rather unwieldy form. A detailed analysis of these equations and their asymptotics is deferred to future work. A few general properties follow from the nature of these equations, however:

1. Assuming small momentum transfer across the vertex, one can Taylor expand the arguments in the $\delta$-functions so that the momentum-conserving $\delta$-function has the argument $\delta\left[k_{\theta_{0}^{h}}^{\prime}\left(\theta_{0}^{h}-\lambda\right)+k_{\theta_{1}^{h}}^{\prime}\left(\theta_{1}^{h}-\theta_{1}^{p}\right)+k_{\theta_{2}^{h}}^{\prime}\left(\theta_{2}^{h}-\theta_{2}^{p}\right)\right]+(1 \leftrightarrow 2)$, and similarly for the energy-conserving $\delta$-function. Given these two conditions, the integral (6) runs over a threedimensional submanifold of the five-dimensional space. This three-dimensional submanifold in turn contains a two-dimensional space corresponding to forward-scattering processes; the matrix element (5) diverges in this subspace but these processes do not relax energy or momentum, and therefore must be excluded from the collision integral by some regularization procedure.

2. The matrix element depends on two dressed charges but not the third. Thus, even if a quasiparticle is neutral under $\hat{q}$, it can relax through this interaction, by scattering off a pair of charged quasiparticles.

3. The decay rates are proportional to the dressed kernel, $\left(K^{\mathrm{dr}}\right)^{2}$, and thus to the strength of interactions in the integrable system.

[1] J. De Nardis and M. Panfil, Journal of Statistical Mechanics: Theory and Experiment 2018, 033102 (2018).

[2] A. Lamacraft, Phys. Rev. A 87, 012707 (2013), URL https://link.aps.org/doi/10.1103/PhysRevA.87.012707.

[3] J. D. Nardis, D. Bernard, and B. Doyon, SciPost Phys. 6, 49 (2019), URL https://scipost.org/10.21468/SciPostPhys. 6.4.049.

[4] A. Cortés Cubero and M. Panfil, arXiv e-prints arXiv:1909.08393 (2019), 1909.08393. 